\title{
Protozoan Parasites of Rodents and Their Zoonotic Significance in Boyer-Ahmad District, Southwestern Iran
}

\author{
Zeinab Seifollahi, Bahador Sarkari, Mohammad Hossein Motazedian, Qasem Asgari, \\ Mohammad Javad Ranjbar, and Samaneh Abdolahi Khabisi \\ Department of Parasitology and Mycology, School of Medicine, Shiraz University of Medical Sciences, Shiraz, Iran \\ Correspondence should be addressed to Bahador Sarkari; sarkarib@sums.ac.ir
}

Received 30 September 2015; Revised 24 January 2016; Accepted 28 January 2016

Academic Editor: Remo Lobetti

Copyright ( 2016 Zeinab Seifollahi et al. This is an open access article distributed under the Creative Commons Attribution License, which permits unrestricted use, distribution, and reproduction in any medium, provided the original work is properly cited.

Backgrounds. Wild rodents are reservoirs of various zoonotic diseases, such as toxoplasmosis, babesiosis, and leishmaniasis. The current study aimed to assess the protozoan infection of rodents in Boyer-Ahmad district, southwestern Iran. Materials and Methods. A total of 52 rodents were collected from different parts of Boyer-Ahmad district, in Kohgiluyeh and Boyer-Ahmad province, using Sherman live traps. Each rodent was anesthetized with ether, according to the ethics of working with animals, and was dissected. Samples were taken from various tissues and stool samples were collected from the contents of the colon and small intestines. Moreover, 2 to $5 \mathrm{~mL}$ of blood was taken from each of the rodents and the sera were examined for antiLeishmania antibodies, by ELISA, or anti-T. gondii antibodies, by modified agglutination test (MAT). DNA was extracted from brain tissue samples of each rodent and PCR was used to identify the DNA of T. gondii. Results. Of the 52 stool samples of rodents studied by parasitological methods, intestinal protozoa infection was seen in 28 cases (53.8\%). From 52 rodents, 19 (36.5\%) were infected with Trichomonas, 10 (19.2\%) with Giardia muris, and $11(21.2 \%)$ with Entamoeba spp. Also, 10 cases (19.2\%) were infected with Blastocystis, 3 (5.8\%) were infected with Chilomastix, 7 (13.5\%) were infected with Endolimax, 1 (1.9\%) was infected with Retortamonas, 3 (5.77\%) were infected with T. gondii, and 6 (11.54\%) were infected with Trypanosoma lewisi. Antibodies to T. gondii were detected in the sera of $5(9.61 \%)$ cases. Results of the molecular study showed T. gondii infection in $3(5.77 \%)$ of the rodents. Findings of this study showed that rodents in Kohgiluyeh and Boyer-Ahmad province, southwestern Iran, are infected with several blood and intestinal parasites; some of them might be potential risks to residents and domestic animals in the region.

\section{Introduction}

Rodents are the most frequent and important mammals on the Earth, because they can adapt themselves to the different locations and environmental changes. These animals live on almost every continent except Antarctica [1]. Rodents are considered as reservoirs of various zoonotic diseases including toxoplasmosis, babesiosis, and leishmaniasis [1-4]. Nevertheless, rodents cannot directly cause disease in humans and disease is mainly transmitted to humans if human is in contact with rodents' feces and secretory materials. Transmission of the zoonotic pathogens to humans can occur via rodent's urine, feces, hair, and saliva [2]. Human activities which change the ecosystem of rodents' living place have an important role in the epidemiology of zoonotic diseases. Given the damage of rodents to humans and economic loss and due to their health importance, parasitological studies on rodents seem necessary [2]. Several studies have been done on parasitic infections of wild rodent in Iran [5-10]. However, due to ecological differences in different areas of the country, the parasitic fauna of the rodents in each ecological setting might be different. This notion justifies new studies on parasitic infection of the rodents in other areas of the country. The current study aimed to assess the parasitic protozoan infections of rodents in Boyer-Ahmad district, southwest Iran.

\section{Materials and Methods}

2.1. The Study Area. Boyer-Ahmad district is located in Kohgiluyeh and Boyer-Ahmad province. The province is located in southwest of Iran with geographical coordinates of $30^{\circ} 40^{\prime} 12^{\prime \prime} \mathrm{N}, 51^{\circ} 36^{\prime} 0^{\prime \prime} \mathrm{E}$. The province has two types 
of tropical and cold climate and Boyer-Ahmad district is located in the cold area. The mean of long-term rain and snow amount is above $600 \mathrm{~mm}$ in this area and a wide area of the county is covered with forests of oak, wild pistachio, and mountain almond. The main professions of the people are agricultural practice and breeding and raising livestock.

2.2. Rodents' Collection and Identification. Considering the map of the study area, 52 rodents were collected from different parts of Boyer-Ahmad County, using Sherman live traps with roasted almonds, as bait, in the summer and autumn of 2014. Different areas of the district, including villages of Kakan, Madvan, Tange Sorkh, Kal Morgah, Mansourabad, and Dehno, were selected for sampling. After transferring to the laboratory, the genus and species of rodents were identified based on morphological characteristics. This was done to subsequently find out the rate of protozoan parasites in each rodent's species.

2.3. Evaluation of Rodents' Protozoan Infection. After transferring the rodents to the laboratory, they were anesthetized with ether and blood samples were taken from their heart. Different rodent parts were carefully examined and necessary samples were prepared. Smears were prepared from rodent liver, spleen, and peripheral blood on glass slides, fixed with methanol, and stained with Giemsa. Then smears were studied by an optical microscope with 100x magnification.

Temporary staining of rodent's stool samples, with Lugol's solution, was done for detection of any protozoan cysts or trophozoites. The samples were also examined with formalin-ethyl acetate sedimentation and zinc sulfate floatation techniques and the obtained materials were observed by conventional light microscope. Smears were also prepared from the rodent stool samples and stained with trichrome. Moreover, smears were prepared from the stool sediments or floated materials, obtained by concentration methods, fixed with methanol, and stained with acid-fast staining to detect coccidia parasites in fecal samples.

2.4. Serological Assessment of Rodents' Sera Samples. Rodents' sera were examined by indirect ELISA for anti-Leishmania antibodies. Moreover, MAT was performed on rodent sera samples, as previously described, to assess anti- $T$. gondii antibodies [11]. Sera were studied in two dilutions of $1: 20$ and $1: 40$ and samples with MAT titer of $1: 40$ or higher were considered as positive.

2.5. Molecular Analysis of Rodents' Tissue Samples. DNA was extracted from brain tissue samples of each rodent, using DNA extraction kit, based on the manufacturer's (YektaTajhiz Azma, Tehran, Iran) instructions. PCR was performed to amplify a 529 bp gene of $T$. gondii, as described by Edvinsson et al. [12]. The two primers used were TOXOF CAG GGA GGA AGA CGA AAGTTG and TOXOR CAG ACA CAG TGC ATC TGG ATT. PCR products were separated in 1.5\% agarose gel and stained with ethidium bromide.

2.6. Statistical Analysis. The statistical analysis was performed with SPSS software (version 16). Chi-square test was

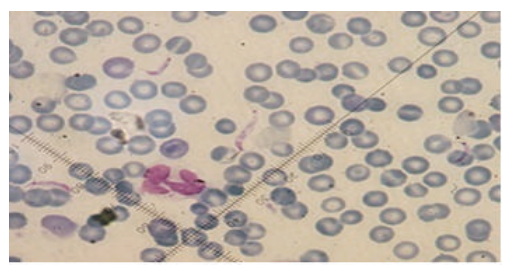

Figure 1: Trypanosoma lewisi in blood smear of the studied rodents, stained with Giemsa (100x).

used to examine the association between rodent's parasitic infections and related studied factors, such as rodent's species, gender, place of collection, and weight.

\section{Results}

A total of 52 rodents were captured during the course of this study, including 25 (48.1\%) Meriones, 15 (28.8\%) Rattus, 10 (19.2\%) Apodemus, 1 (1.9\%) Calomyscus, and 1(1.9\%) Arvicola. Among the captured rodents, 28 (53.8\%) were males and 24 (46.2\%) were females.

Of the 52 feces samples of rodents, examined by parasitological methods, 37 (71.1\%) were infected with at least one protozoan parasite, whereas $15(28.8 \%)$ of the rodents were not infected with any intestinal protozoan parasites. From 52 rodents, 19 (36.5\%) were infected with Trichomonas, 10 (19.2\%) with G. muris, 11 (21.2\%) with Entamoeba, 10 (19.2\%) with Blastocystis, 3 (5.8\%) with Chilomastix, 7 (13.5\%) with Endolimax, and 1 (1.9\%) was infected with Retortamonas. Regarding the rodents' infection with blood and tissue protozoa, $3(5.77 \%)$ were infected with T. gondii and 6 (11.54\%) with Trypanosoma lewisi (Figure 1). Anti-Leishmania antibodies were detected in the sera of $8(15.34 \%)$ of the rodents; among them were 6 rodents which were also infected with Trypanosoma lewisi. No Leishmania parasites were observed in the impression smears of liver or spleen of the seropositive rodents. Figure 2 shows a few of intestinal protozoa detected in trichrome-stained samples of rodents' feces.

Multiple infections were seen in 19 out of 52 (36.5\%) rodents. Simultaneous infection with Trichomonas and Entamoeba was seen in 5.8\%, Trichomonas and G. muris in 1.9\%, G. muris and Trypanosoma in 1.9\%, Blastocystis and G. muris in $1.9 \%$, Trichomonas and Blastocystis in 1.9\%, and Entamoeba and Blastocystis in $1.9 \%$ of the rodents. Also, simultaneous infection with Blastocystis, Trypanosoma, and Endolimax was observed in $1.9 \%$ of the rodents.

In this study, $54.2 \%$ of rodents, infected with intestinal protozoa, were female and $45.8 \%$ were male. Statistical analysis showed no significant correlation between various protozoa and gender of the rodents $(P>0.05)$.

The highest rates of infection with G. muris (70\%), Trichomonas (36.8\%), Endolimax (71.4\%), Trypanosoma (100\%), and Blastocystis (60\%) were seen in Rattus genus and the highest infection with Entamoeba (54.5\%) was seen in the genus Meriones. Statistical analysis showed significant correlation between protozoa infection and the rodent's genus $(P<$ 0.05). Table 1 shows the distribution of protozoan infections according to the rodent genus. 

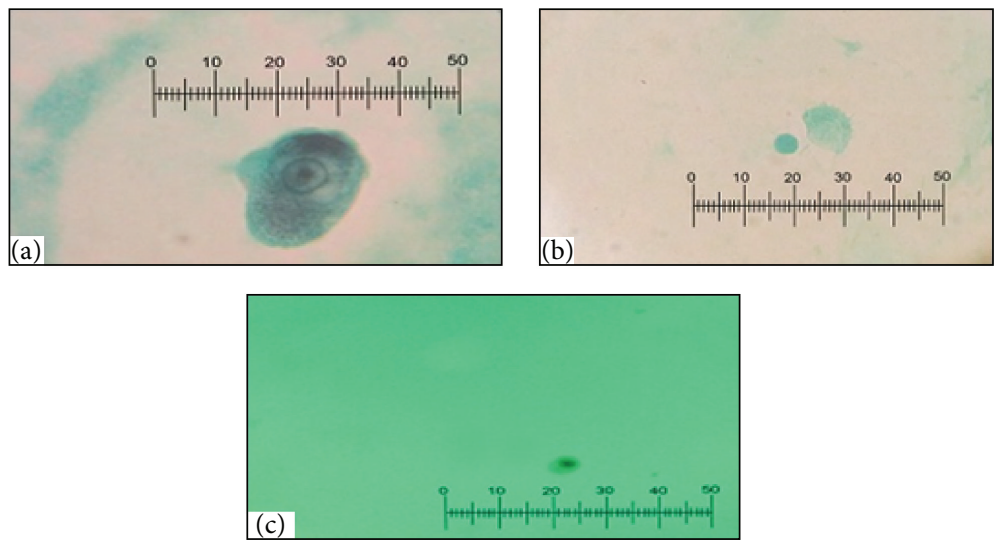

FIgURE 2: Protozoa in stool samples of the rodents, stained with trichrome. (a) Entamoeba trophozoite, (b) Trichomonas trophozoite, and (c) Endolimax trophozoite (100x).

TABLE 1: Distribution of protozoan infection according to genus of the studied rodents.

\begin{tabular}{|c|c|c|c|c|c|c|c|c|c|c|c|c|}
\hline & \multicolumn{2}{|c|}{ Rattus } & \multicolumn{2}{|c|}{ Meriones } & \multicolumn{2}{|c|}{ Calomyscus } & \multicolumn{2}{|c|}{ Apodemus } & \multicolumn{2}{|c|}{ Arvicola } & \multicolumn{2}{|c|}{ Total } \\
\hline & Number & $\%$ & Number & $\%$ & Number & $\%$ & Number & $\%$ & Number & $\%$ & Number & $\%$ \\
\hline G. muris & 7 & 70 & 2 & 20 & 0 & 0 & 1 & 10 & 0 & 0 & 10 & 19.23 \\
\hline Trichomonas & 7 & 36.8 & 4 & 21.1 & 1 & 5.3 & 7 & 36.8 & 0 & 0 & 19 & 36.5 \\
\hline Blastocystis & 6 & 60 & 2 & 20 & 1 & 10 & 1 & 10 & 0 & 0 & 10 & 19.23 \\
\hline Entamoeba & 4 & 36.4 & 6 & 54.5 & 0 & 0 & 1 & 1.9 & 0 & 0 & 11 & 21.1 \\
\hline Chilomastix & 1 & 33.3 & 1 & 33.3 & 0 & 0 & 1 & 33.3 & 0 & 0 & 3 & 5.8 \\
\hline Endolimax & 5 & 71.4 & 1 & 14.3 & 0 & 0 & 1 & 33.3 & 0 & 0 & 7 & 13.46 \\
\hline Retortamonas & 0 & 0 & 0 & 0 & 0 & 0 & 1 & 100 & 0 & 0 & 1 & 1.92 \\
\hline Trypanosoma & 6 & 100 & 0 & 0 & 0 & 0 & 0 & 0 & 0 & 0 & 6 & 11.53 \\
\hline T. gondii & 0 & 0 & 1 & 33.3 & 1 & 33.3 & 1 & 33.3 & 0 & 0 & 3 & 508 \\
\hline
\end{tabular}

Findings of the molecular study showed T. gondii infection in $3(5.77 \%)$ rodents; two male and one female. Rodents infected with T. gondii were from Apodemus, Meriones, and Calomyscus genus. Figure 3 shows PCR products of DNA, isolated from rodents' brain tissue.

No cases of coccidial infection were seen in any of the fecal samples when the samples were evaluated by modified acid-fast staining method.

\section{Discussion}

Rodents are considered as reservoirs for a few of helminthic and protozoan parasites $[1,2]$. Among the protozoa parasites of rodents is T. gondii which is common in rodents and these animals can behave as natural reservoir for this protozoa. Evaluation of $T$. gondii infection in rodents, as the main pray for cat, with regards to the role of cat in spreading of T. gondii oocyst in the environment, is important [13]. Rate of Toxoplasma infection in rodents is different based on ecological status of a given area. In the current study, T. gondii infection was common protozoa of the studied rodents. Saki and Khademvatan reported a prevalence rate of $6 \%$ for $T$. gondii in rodents of Ahvaz district, south of Iran [14]. Study of Mercier et al. in 2013, assessing 766 rodents in Niamey district of Niger, revealed Toxoplasma infection in 1.96\%

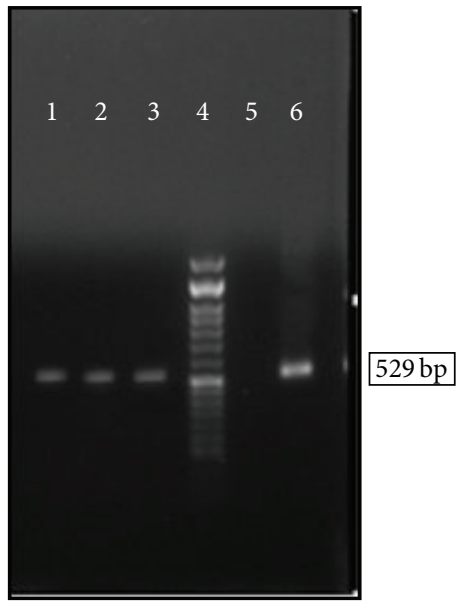

FIGURE 3: The PCR product of the DNA of T. gondii isolated from the rodents brain tissues. Lanes 1-3, samples isolated from rodents' brain tissue; lane 4, 50 bp DNA Ladder; lane 5, negative control; lane 6 , positive control (tachyzoite prepared from mice peritonea).

of the studied rodents [15]. In the current study, T. gondii infection was found in Apodemus, Meriones, and Calomyscus genus and, to the best of our knowledge, this is the first report 
of molecular detection of T. gondii infection in Calomyscus from Iran.

Another important parasitic infection that rodents have an important role in its transmission, as reservoirs, is leishmaniasis. A large number of species of rodents have been identified as reservoir of cutaneous leishmaniasis in Iran [35]. Mohebali et al. reported the infection of different species of the rodents, including Rhombomys opimus, Meriones libycus, Tatera indica, and Meriones hurrianae with L. major [16]. Rassi et al. reported that Meriones libycus is the main reservoir of cutaneous leishmaniasis in Fars province, southern Iran [17]. In the present study, Leishmania infection was not detected in any of the studied rodents. The reason for this is that leishmaniasis is mainly seen in tropical and subtropical areas of Iran, while Boyer-Ahmad district is located in cold and mountainous region of the country and is not considered as an endemic focus of cutaneous leishmaniasis. Although visceral leishmaniasis is not uncommon in this district, its reservoirs are dogs, carnivores, or properly cats, rather than rodents [18-20].

Rodents are frequently infected with intestinal protozoa and may act as reservoir for a few of them. In the current study, intestinal protozoa including Trichomonas, Entamoeba, and G. muris were detected in the studied rodents. Rate of Giardia infection, as the main intestinal protozoa, in rodents was found to be $14.6 \%$ in Al Hindi and Abu-Haddaf study in 2013 in Palestine, 96.3\% in Microtus and 48.3\% in Apodemus species in Poland, 2.5\% in the study by Rasti and colleagues in 2000 in Kashan, Iran, and 2.7\% in the study by Kia and colleagues in 2001 in Ahvaz, south of Iran [7, 21-23]. In the current study, rate of G. muris infection in the rodents was relatively high. Further study is needed to compare the genotype of these protozoa, isolated from the rodents with the human isolates.

Infection with blood protozoa, Trypanosoma, was common in the studied rodents in our study. Lower rate of infection (10\%) with this parasite has been reported in Kia et al. study in Ahvaz, south of Iran [7], whereas higher rates of infection have been reported from Brazil (21.7\%) and India $(82.3 \%)[24,25]$.

In this study, infection with intestinal coccidia was not found in the rodents, while other studies have reported coccidia infection in these animals [21]. This difference could be due to the differences in climatic conditions in the studied areas, and also nutritional or habitat preferences of the rodents.

Taken together, findings of the current study revealed that rodents in Kohgiluyeh and Boyer-Ahmad province, in southwest of Iran, are infected with many intestinal and blood protozoa. Some of these protozoa may be potential risks to the residents and domestic animals in the region. High prevalence of intestinal protozoan infections in the rodents might be linked to the unsafe disposal of human waste and also use of human and animal fertilizers in the area.

\section{Conflict of Interests}

The authors declare that there is no conflict of interests regarding the publication of this paper.

\section{Acknowledgments}

The results described in this paper were part of MSc thesis of Zeinab Seifollahi. The study was financially supported by the office of vice-chancellor for research of Shiraz University of Medical Sciences (Grant No. 7322-93).

\section{References}

[1] B. G. Meerburg, G. R. Singleton, and A. Kijlstra, "Rodent-borne diseases and their risks for public health," Critical Reviews in Microbiology, vol. 35, no. 3, pp. 221-270, 2009.

[2] B. G. Meerburg, "Rodents are a risk factor for the spreading of pathogens on farms," Veterinary Microbiology, vol. 142, no. 3-4, pp. 464-465, 2010.

[3] M. H. Davami, M. H. Motazedian, M. Kalantari et al., "Molecular survey on detection of Leishmania infection in rodent reservoirs in Jahrom District, Southern Iran," Journal of ArthropodBorne Diseases, vol. 8, no. 2, pp. 139-146, 2014.

[4] B. Pourmohammadi, M. H. Motazedian, and M. Kalantari, "Rodent infection with Leishmania in a new focus of human cutaneous leishmaniasis, in northern Iran," Annals of Tropical Medicine and Parasitology, vol. 102, no. 2, pp. 127-133, 2008.

[5] D. Mehrabani, M. H. Motazedian, A. Oryan, Q. Asgari, G. R. Hatam, and M. Karamian, "A search for the rodent hosts of Leishmania major in the Larestan region of southern Iran: demonstration of the parasite in Tatera indica and Gerbillus sp., by microscopy, culture and PCR," Annals of Tropical Medicine and Parasitology, vol. 101, no. 4, pp. 315-322, 2007.

[6] S. Gholami, H. F. Motevali, E. Moabedi, and S. Shahabi, "Study of helmintic intestinal parasites in the rodents from the rural and central regions of Mazandaran province in the years 1997 to 1999," Journal of Mazandaran University of Medical Sciences, vol. 12, no. 35, pp. 67-75, 2002.

[7] E. Kia, M. Homayouni, A. Farahnak, M. Mohebali, and S. Shojai, "Study of endoparasites of rodents and their zoonotic importance in Ahvaz, South West Iran," Iranian Journal of Public Health, vol. 30, pp. 49-52, 2001.

[8] E. B. Kia, E. Shahryary-Rad, M. Mohebali et al., "Endoparasites of rodents and their zoonotic importance in Germi, DashteMogan, Ardabil Province, Iran," Iranian Journal of Parasitology, vol. 5, no. 4, pp. 15-20, 2010.

[9] S. M. Sadjjadi and J. Massoud, "Helminth parasites of wild rodents in Khuzestan province, south west of Iran," Journal of Veterinary Parasitology, vol. 13, no. 1, pp. 55-56, 1999.

[10] A. Salehabadi, G. Mowlavi, and S. M. Sadjjadi, "Human infection with Moniliformis moniliformis (Bremser 1811) (Travassos 1915) in Iran: another case report after three decades," VectorBorne and Zoonotic Diseases, vol. 8, pp. 101-104, 2008.

[11] B. Sarkari, Q. Asgari, N. Bagherian et al., "Molecular and Serological evaluation of Toxoplasma gondii infection in reared turkeys in Fars Province, Iran," Jundishapur Journal of Microbiology, vol. 7, no. 7, Article ID e11598, 2014.

[12] B. Edvinsson, S. Jalal, C. E. Nord, B. S. Pedersen, and B. Evengård, "DNA extraction and PCR assays for detection of Toxoplasma gondii," APMIS, vol. 112, no. 6, pp. 342-348, 2004.

[13] C. Gotteland, Y. Chaval, I. Villena et al., "Species or local environment, what determines the infection of rodents by Toxoplasma gondii?” Parasitology, vol. 141, no. 2, pp. 259-268, 2014. 
[14] J. Saki and S. Khademvatan, "Detection of Toxoplasma gondii by PCR and mouse bioassay in rodents of Ahvaz District, Southwestern Iran," BioMed Research International, vol. 2014, Article ID 383859, 5 pages, 2014.

[15] A. Mercier, M. Garba, H. Bonnabau et al., "Toxoplasmosis seroprevalence in urban rodents: a survey in Niamey, Niger," Memorias do Instituto Oswaldo Cruz, vol. 108, no. 4, pp. 399407, 2013.

[16] M. Mohebali, E. Javadian, M. R. Yaghoobi-Ershadi, A. A. Akhavan, H. Hajjaran, and M. R. Abaei, "Characterization of Leishmania infection in rodents from endemic areas of the Islamic Republic of Iran," Eastern Mediterranean Health Journal, vol. 10, no. 4-5, pp. 591-599, 2004.

[17] Y. Rassi, M. Jalali, E. Javadian, and M. Moatazedian, "Confirmation of Meriones libycus (Rodentia; Gerbillidae) as the main reservoir host of zoonotic cutaneous leishmaniasis in arsanjan, fars province, South of Iran (1999-2000)," Iranian Journal of Public Health, vol. 30, no. 3-4, pp. 143-144, 2001.

[18] B. Sarkari, N. Pedram, M. Mohebali et al., "Seroepidemiological study of visceral leishmaniasis in Booyerahmad district, southwest Islamic Republic of Iran," Eastern Mediterranean Health Journal, vol. 16, no. 11, pp. 1133-1136, 2010.

[19] G. R. Hatam, S. J. Adnani, Q. Asgari et al., "First report of natural infection in cats with Leishmania infantum in Iran," VectorBorne and Zoonotic Diseases, vol. 10, no. 3, pp. 313-316, 2010.

[20] B. Sarkari, G. R. Hatam, S. J. Adnani, and Q. Asgari, "Seroprevalence of feline leishmaniasis in areas of Iran where Leishmania infantum is endemic," Annals of Tropical Medicine and Parasitology, vol. 103, no. 3, pp. 275-277, 2009.

[21] A. I. Al Hindi and E. Abu-Haddaf, "Gastrointestinal parasites and ectoparasites biodiversity of Rattus rattus trapped from Khan Younis and Jabalia in Gaza strip, Palestine," Journal of the Egyptian Society of Parasitology, vol. 43, no. 1, pp. 259-268, 2013.

[22] S. Rasti, I. Moubedi, R. Dehghani, and A. Drodgar, "The survey of gastrointestinal helminths of mice in Kashan," Journal of the Faculty of Veterinary Medicine, University of Tehran, vol. 55, no. 4, pp. 57-59, 2000.

[23] A. Bajer, M. Bednarska, A. Pawełczyk, J. M. Behnke, F. S. Gilbert, and E. Sinski, "Prevalence and abundance of Cryptosporidium parvum and Giardia spp. in wild rural rodents from the Mazury Lake District region of Poland," Parasitology, vol. 125, no. 1, pp. 21-34, 2002.

[24] R. Laha, H. Hemaprasanth, and D. Bhatta-Charya, "Observations on prevalence of Trypanosoma lewisi infection in wild rats and a trial on its adaptation in unnatural host," Journal of Parasitology and Applied Animal Biology, vol. 6, pp. 5-8, 1997.

[25] P. M. Linardi and J. R. Botelho, "Prevalence of Trypanosoma lewisi in Rattus norvegicus from Belo Horizonte, State of Minas Gerais, Brazil," Memórias do Instituto Oswaldo Cruz, vol. 97, no. 3, pp. 411-414, 2002. 

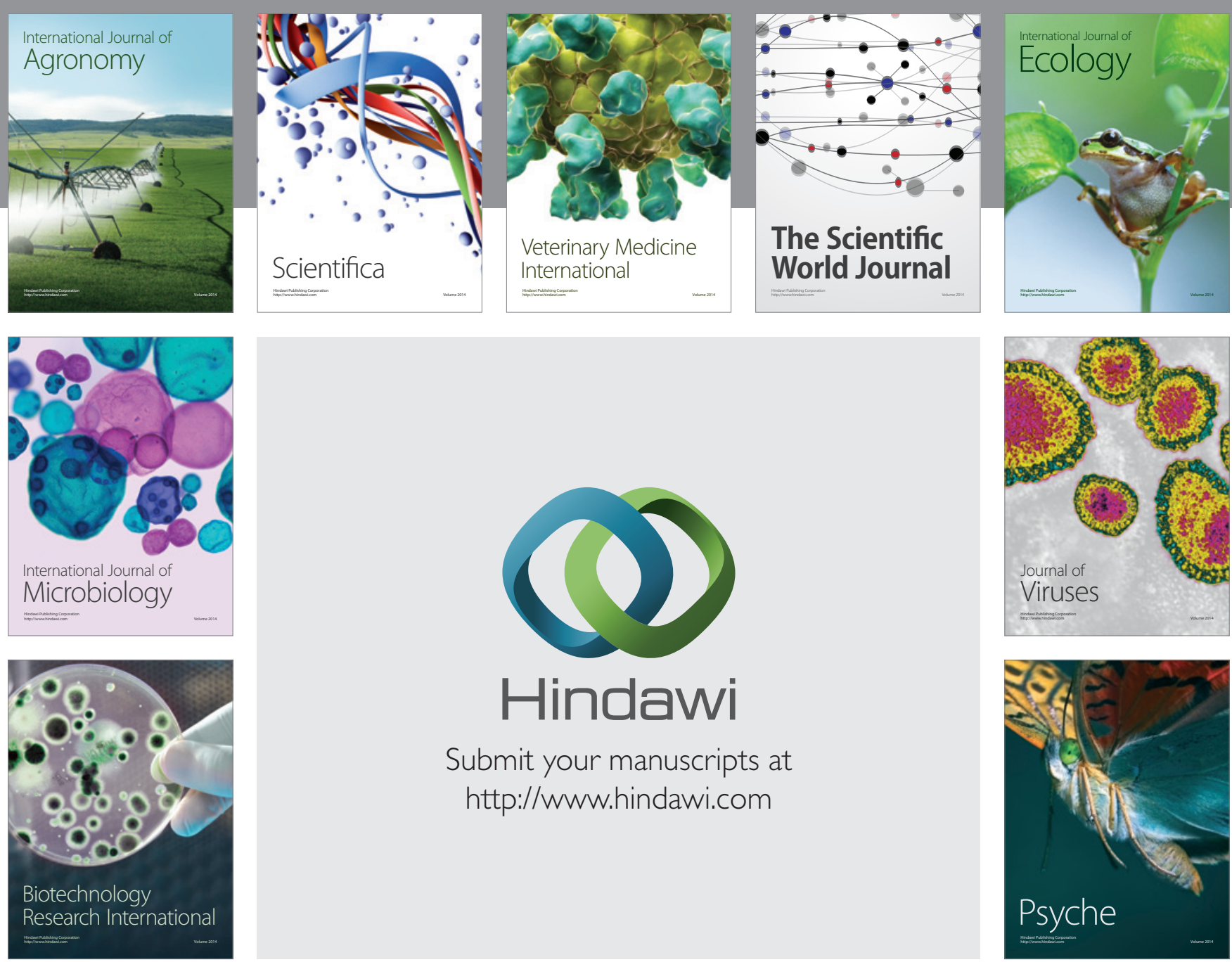

Submit your manuscripts at

http://www.hindawi.com
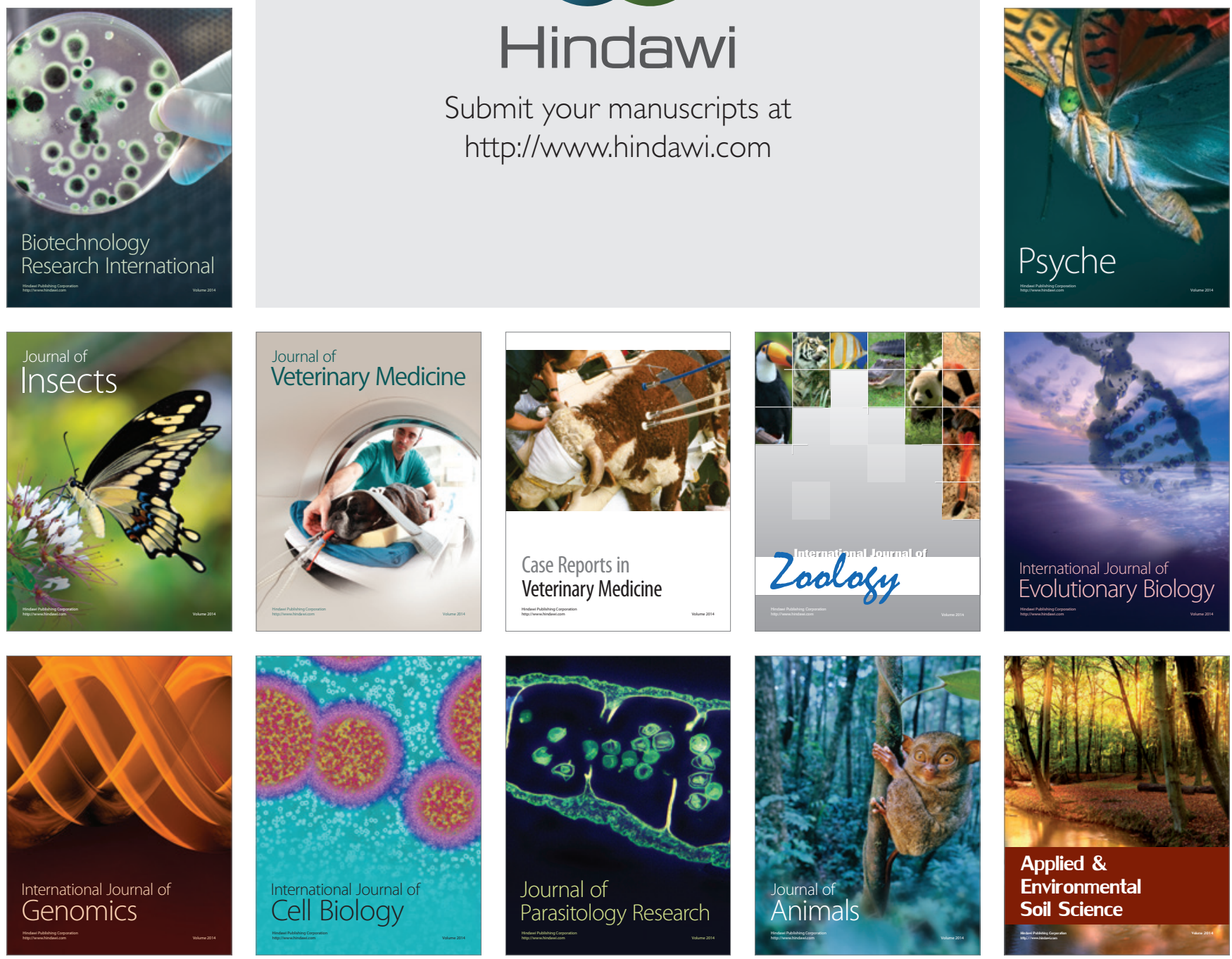